\title{
Improving Airfoil Drag Prediction
}

\author{
Giridhar Ramanujam \\ ${ }^{\dagger}$ Energy Research Centre of the Netherlands(ECN), 1755LE, Petten, The Netherlands \\ ${ }^{\ddagger}$ University of Twente, 7500 AE, Enschede, The Netherlands \\ Hüseyin Özdemir ${ }^{\S}$ \\ Energy Research Centre of the Netherlands(ECN), 1755LE, Petten, The Netherlands \\ H.W.M.Hoeijmakers $₫$ \\ University of Twente, 7500AE, Enschede, The Netherlands
}

\begin{abstract}
An improved formulation of drag estimation for thick airfoils is presented. Drag underprediction in XFOIL like viscous-inviscid interaction methods can be quite significant for thick airfoils used in wind turbine applications (up to $30 \%$ as seen in the present study). The improved drag formulation predicts the drag accurately for airfoils with reasonably small trailing edge thickness. The derivation of drag correction is based on the difference between the actual momentum loss thickness based on free stream velocity and the one based on the velocity at the edge of the boundary layer. The improved formulation is implemented in the most recent version of XFOIL and RFOIL (an aerodynamic design and analysis method based on XFOIL, developed by a consortium of ECN, NLR and TU Delft after ECN acquired the XFOIL code. After 1996, ECN maintained and improved the tool.) and the results are compared with experimental data, results from commercial CFD methods like ANSYS CFX and other methods like DTU-AED EllipSys2D and CENER WMB. The improved version of RFOIL shows good agreement with experimental data.
\end{abstract}

\section{Nomenclature}

$\alpha$

$\Delta \theta$

$\delta$

$\delta^{*}$

$\infty$

$\rho$

$\theta$

$\xi, \eta$

A, B

air foil

$c$

$C_{\tau_{E Q}}$

$c_{d}$

$c_{l}$

$D$

$e$
Angle of attack

Error in $\theta$

Boundary layer thickness

Boundary layer displacement thickness

Subscript for incident free stream condition

Density of fluid

Boundary layer momentum thickness

Streamline space coordinates

$G-\beta$ equilibrium locus coefficients

Subscript for airfoil parameters

Airfoil chord length

Equilibrium maximum shear stress coefficient

Sectional drag coefficient

Sectional lift coefficient

Drag

Subscript for boundary layer edge condition

\footnotetext{
${ }^{\dagger}$ Researcher, Wind Energy Unit, Westerduinweg 3, ramanujam@ecn.nl, Member AIAA

${ }^{\ddagger}$ Former M.Sc. Student, Sustainable Energy Technology, giridhar.ramanujam@gmail.com

§Researcher, Wind Energy Unit, Westerduinweg 3, h.ozdemir@ecn.nl, Member AIAA

TProfessor, Department of Mechanical Engineering, Engineering Fluid Dynamics, PO Box 217, h.w.m.hoeijmakers@utwente.nl, Senior Member AIAA
} 


effective
$f p$
$g$
$H$
$H^{*}$
$H_{1}$
$H_{k}$
$h_{T E}$
$L$
$M_{\infty}$
$N_{c r i t}$
$R e_{\theta}$
$R e_{c}$
$R F O I L$
$u$
$U_{\infty}$
$U_{e}$
$w a k e$
$x, y$
$x=\infty$
$x_{C_{\tau_{E} Q}}$
$X F O I L$

Subscript for overall domain parameters

Subscript for flat plate

Correction factor for mass flow shape factor

Shape factor

Kinetic energy shape factor

Mass flow shape factor

Kinematic shape factor

Airfoil trailing edge thickness

Length scale reference parameter

Free stream Mach number

Critical amplification factor

Momentum thickness Reynolds number

Chord length based Reynolds number

Subscript for RFOIL parameters

Velocity

Incident free stream velocity

Boundary layer edge velocity

Subscript for wake parameters

Cartesian space coordinates

Subscript for infinity downstream location

Equilibrium shear stress coefficient multiplier in wake

Subscript for XFOIL parameters

\section{Introduction}

ETHODs for accurate estimation of drag for airfoils is an important criterion for the analysis and design of airfoils. Since for an airfoil, the drag is usually two orders of magnitude smaller than lift, even small errors in drag values can cause a significant change in airfoil performance (lift to drag ratio). Thick airfoils are commonly used in wind turbine blades with the thickness varying from $15 \%$ of chord at the tip section to about $50 \%$ of chord at the root section. It is thus important to have an accurate prediction for drag in order to determine the performance of the wind turbine. With the trend of size of wind turbines increasing, thicker airfoils are becoming more and more important as the structural requirements need to be satisfied while maintaining optimal aerodynamic performance. Present day drag estimation models appear to underpredict the drag by a significant margin ranging from $10 \%$ for thin airfoils to as high as $30 \%$ or more for thick airfoils with negligible trailing edge thickness $\left(h_{T E}<3 \%\right.$ of chord) as can be seen in the results of the present study. In this paper, the method currently used for the calculation of drag for airfoils is studied in detail and the cause of inaccuracy is analysed. The limitations of the present method are also investigated in order to explain why it gives acceptable results for a certain range of airfoil types. Finally, a correction is proposed to improve the prediction of drag and the results based on the correction are discussed.

\section{Aerodynamic Methods}

For the analysis presented in this paper, $\mathrm{XFOIL}^{1}$ and $\mathrm{RFOIL}^{2}$ have been used. XFOIL ${ }^{\mathrm{a}}$ is a viscousinviscid interaction method for predicting flow about airfoils developed by Mark Drela at MIT. It utilizes a linear-vorticity panel method with Karman-Tsien compressibility correction for analysis in direct and mixedinverse modes. Source distributions superimposed on the airfoil and wake permit modelling of viscous layer effects on potential flow results. A two-equation lagged dissipation integral method is used to represent the viscous layers. Both laminar and turbulent flows are treated with an $e^{N}$-type amplification formulation determining the transition point. The boundary layer and transition equations are solved simultaneously with the inviscid flow field by a global Newton method. The procedure is especially suitable for rapid analysis of low Reynolds number flows around airfoils with transitional separation bubbles. RFOIL is a modified version of XFOIL featuring an improved prediction for the maximum lift coefficient and includes a method for predicting the effect of rotation on airfoil characteristics. Regarding the maximum lift in particular,

$\overline{\text { a } \quad \text { Refers to XFOIL } 6.99 \text { unless stated otherwise }}$ 
numerical stability improvements were obtained by using the Schlichting velocity profiles for the turbulent boundary layer, instead of Swafford's velocity profiles incorporated in XFOIL. Furthermore, the shear lag coefficient in Green's lag entrainment equation of the turbulent boundary layer model was adjusted based on the shape factor of the boundary layer for deviation from the equilibrium flow observed at high values of the shape factor. From the results of the validation, $10 \%$ under-prediction has been found for the drag. ${ }^{2}$

\section{Momentum Thickness and Drag - Current Formulation}

The currently used formulation for estimation of the drag on an airfoil is based on flat plate boundary layer theory. The momentum conservation in $x$-direction (direction of incident free stream) yields the drag on a body immersed in the flow. The expression for drag of a flat plate ${ }^{3,4}$ is given in terms of momentum thickness as follows,

$$
D_{f p}=\rho U_{\infty}^{2}\left[\theta_{\infty}\right]_{x=\infty},
$$

where the subscript $x=\infty$ indicates value of the parameter at the far wake of the airfoil and $\theta_{\infty}$ is the momentum thickness (for incompressible flow) based on free-stream velocity (indicated by subscript $\infty$ ) given by,

$$
\theta_{\infty}=\int_{0}^{\delta} \frac{u}{U_{\infty}}\left(1-\frac{u}{U_{\infty}}\right) d y
$$

The above expression of drag involves the following assumptions,

- Incident free stream velocity $\left(U_{\infty}\right)$ is in $x$-direction

- $U_{\infty}$ is a constant in space and time coordinates

- The velocity outside the boundary layer (the edge velocity) equals $U_{\infty}$. i.e. zero pressure gradient along the flow

- The flow is in steady state

- The flow is incompressible

In dimensionless form, the drag coefficient is given by,

$$
c_{d}=\frac{2\left[\theta_{\infty}\right]_{x=\infty}}{L}
$$

where $L$ is a typical length scale reference parameter. For airfoils, the chosen length scale parameter is the chord length.

In RFOIL (as in XFOIL), the drag coefficient is calculated as given in Eq.(3) but the definition of momentum thickness is different. The momentum thickness is given in terms of stream-wise coordinates instead of Cartesian coordinates as defined in Eq.(2) and the reference velocity is the velocity at the edge of the boundary layer $\left(U_{e}\right)$ instead of the free stream velocity $\left(U_{\infty}\right)$. The edge velocity $\left(U_{e}\right)$ on the airfoil surface is obtained by coupling the boundary layer equations to the potential flow equations describing inviscid, irrotational flow in the outer region using a strong interaction scheme. The expression for momentum thickness in RFOIL is as follows,

$$
\theta_{e}=\int_{0}^{\delta} \frac{u}{U_{e}}\left(1-\frac{u}{U_{e}}\right) d \eta
$$

and the drag coefficient is given by,

$$
c_{d_{\text {RFOIL }}}=\frac{2\left[\theta_{e}\right]_{x=\infty}}{c},
$$

where the subscript $e$ denotes the momentum thickness calculated by using $U_{e}$ as reference velocity. 
The two definitions of the drag coefficient are not the same. Eq.(3) gives the force in $x$-direction, which is the definition of drag, whereas Eq.(5) gives the force along $\xi$-direction (streamwise direction) which may not necessarily be equal to the actual drag force on the airfoil. But this assumption is reasonable at a location far downstream of the airfoil, where the flow is almost in the free-stream direction. Thus, in the far wake of an airfoil, the streamwise and cartesian coordinates are almost coincident.

$$
\xi \sim x \quad, \quad \eta \sim y .
$$

However, the use of $U_{e}$ instead of $U_{\infty}$ as the reference velocity in the momentum thickness calculation would cause a significant difference in the estimation of the drag. In the next section, this difference is analysed in detail and a correction is proposed.

\section{Drag Correction}

The relation for drag follows from momentum conservation along incident flow direction ( $x$-direction). However, there is a significant difference between a flat plate and an airfoil in terms of the pressure distribution. There exists a pressure gradient along and normal to the surface of the airfoil which is a result of the airfoil being of finite thickness as opposed to a flat plate which is considered to have zero thickness. There is also an important effect of the angle of attack which is zero for the flat plate theory. As a consequence, the outer region (outside the boundary layer) which is governed by the potential flow equations has a significant pressure gradient in the $\eta$-direction (normal to streamline direction). This pressure gradient causes the velocity outside the boundary layer to vary in the $\eta$-direction. For the analysis, we assume that the velocity outside the boundary layer is constant in $\eta$-direction and hence we can neglect the viscous effects in that region. This assumption is only valid when $U_{e}=U_{\infty}$, which is the flat plate case. For an airfoil, the assumption that velocity outside the boundary layer is $U_{e}$ at all points in the normal direction results in an under prediction of the integral quantities displacement thickness $\left(\delta^{*}\right)$ and momentum thickness $(\theta)$. This also leads to an over prediction of $U_{e}$. In effect, the under prediction of $\theta$ reduces the predicted drag and over prediction of $U_{e}$ increases lift. This behaviour is very commonly seen in viscous-inviscid interaction methods like XFOIL and RFOIL.

To calculate the error in drag, we need to estimate the error in the momentum thickness $(\Delta \theta)$ at the end of wake (taken to be one chord length downstream of the airfoil in XFOIL and RFOIL). $\Delta \theta$ is the difference between the actual momentum loss thickness $\theta_{\infty}$ which is based on free stream velocity $\left(U_{\infty}\right)$ and the predicted momentum loss thickness $\theta_{e}$ which is based on calculated value of velocity at the edge of the boundary layer $\left(U_{e}\right)$. It is important to note that this error $\Delta \theta$ is a deviation of the physical model used

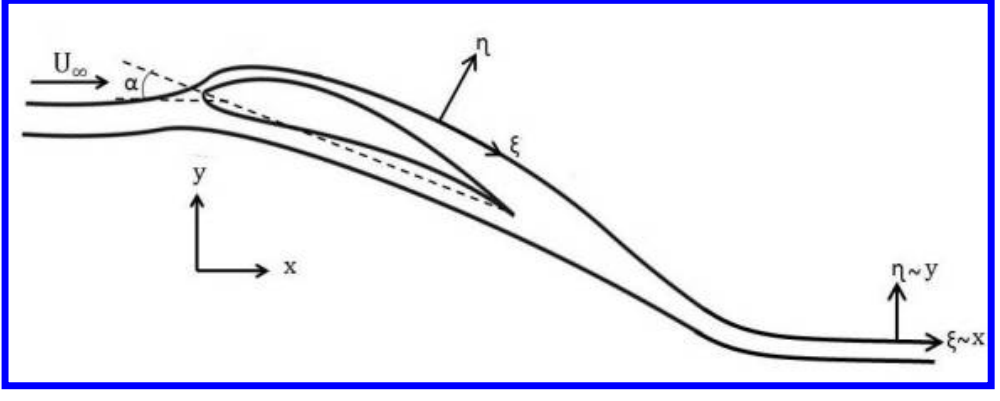

Figure 1: Cartesian $(x, y)$ and Streamwise $(\xi, \eta)$ directions on an airfoil by viscous-inviscid interaction methods from the real physical process, not an error of a numerical origin. For the following part of this section, all boundary layer variables are taken to be at the end of wake.

$$
\Delta \theta=\theta_{\infty}-\theta_{e} .
$$

As mentioned earlier in Eq.(6), $\eta \sim y$ at the end of wake. This allows us to write the expression of $\theta_{e}$ as a integration in $y$-direction. Also the limits of integration can be taken as the boundary layer thickness $(\delta)$, from 0 to $\delta$ (the lower limit is taken zero in XFOIL and RFOIL since the wake is considered as symmetric and the initial values of the boundary layer variables are taken as the sum of upper and lower airfoil surface trailing edge values). Based on this, we have 


$$
\begin{aligned}
\Delta \theta & =\int_{0}^{\delta} \frac{u}{U_{\infty}}\left(1-\frac{u}{U_{\infty}}\right) d y-\int_{0}^{\delta} \frac{u}{U_{e}}\left(1-\frac{u}{U_{e}}\right) d y \\
& =\int_{0}^{\delta}\left(\left(\frac{u}{U_{\infty}}-\frac{u}{U_{e}}\right)-\left(\frac{u^{2}}{U_{\infty}^{2}}-\frac{u^{2}}{U_{e}^{2}}\right)\right) d y \\
& =\left(\frac{U_{e}}{U_{\infty}}-1\right) \int_{0}^{\delta}\left(\frac{u}{U_{e}}-\left(\frac{U_{e}}{U_{\infty}}+1\right) \frac{u^{2}}{U_{e}^{2}}\right) d y \\
& =\left(\frac{U_{e}}{U_{\infty}}-1\right)\left(\theta_{e}-\frac{U_{e}}{U_{\infty}} \int_{0}^{\delta} \frac{u^{2}}{U_{e}^{2}} d y\right) \\
& =\left(\frac{U_{e}}{U_{\infty}}-1\right)\left(\theta_{e}+\frac{U_{e}}{U_{\infty}} \int_{0}^{\delta}\left(1-\frac{u^{2}}{U_{e}^{2}}-1\right) d y\right) \\
& =\left(\frac{U_{e}}{U_{\infty}}-1\right)\left(\theta_{e}+\frac{U_{e}}{U_{\infty}}\left(\delta_{e}^{*}+\theta_{e}-\delta\right)\right),
\end{aligned}
$$

where $\delta_{e}^{*} \equiv \int_{0}^{\delta}\left(1-\frac{u}{U_{e}}\right) d y$. Since $H_{1} \equiv \frac{\delta-\delta_{e}^{*}}{\theta_{e}}$ in XFOIL and RFOIL, we have

$$
\Delta \theta=\theta_{e}\left(1-\frac{U_{e}}{U_{\infty}}\right)\left(\frac{U_{e}}{U_{\infty}}\left(H_{1}-1\right)-1\right)
$$

where the value of $H_{1}$ is obtained using the correlation from Green ${ }^{5}$ simplified by Drela. ${ }^{6}$

$$
H_{1}=3.15+\frac{1.72}{H_{k}-1} \text {. }
$$

This expression is based on experimental results and correlates $\delta$ to $\delta_{\infty}^{*}$ and $\theta_{\infty}$ as $H_{1} \equiv \frac{\delta-\delta_{\infty}^{*}}{\theta_{\infty}}$. Since $\delta_{\infty}^{*}$ and $\theta_{\infty}$ are larger than $\delta_{e}^{*}$ and $\theta_{e}$ respectively (as evidenced from drag under-prediction and lift over-prediction in XFOIL and RFOIL), using the edge velocity based values will result in an over prediction of $H_{1}$. As seen from Eq.(9), the value of $H_{1}$ depends only on the value of kinematic shape factor $\left(H_{k}\right)$, which for incompressible flow is the same as the shape factor $H\left(\equiv \delta^{*} / \theta\right)$. The rate of change of $H_{1}$ with respect to $H$ becomes extremely high for values of $H$ tending to unity $(H \rightarrow 1$, see figure 2). Thus in the far wake, where the value of $H$ is close to unity, the value of $H_{1}$ is very sensitive to the prediction of $H$ and even small changes in the boundary layer model can cause a significant difference in the results. This difference is seen in the results of RFOIL compared to XFOIL. In the next section, this difference in prediction is explored in order to obtain a generalised expression for the $\theta$ correc-

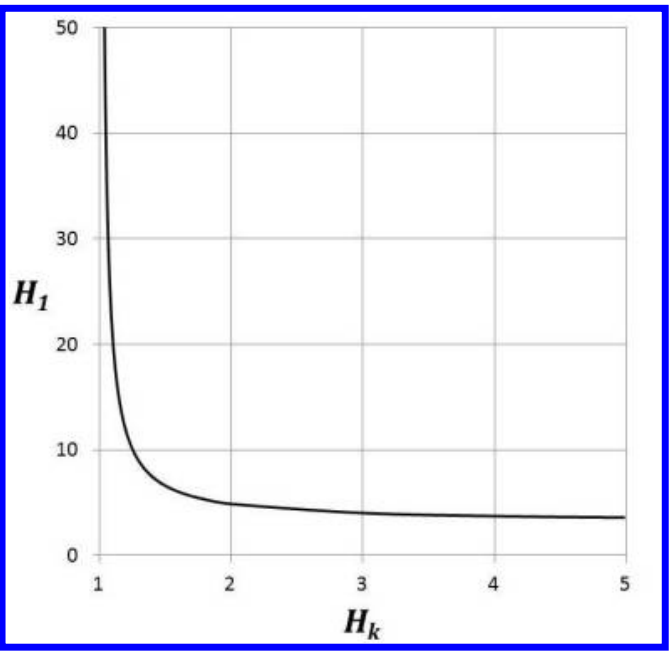

Figure 2: $H_{1}-H_{k}$ correlation from Green ${ }^{5}$ tion.

In order to correct the value of $H_{1}$, we introduce a constant $g$ as a multiplier to $H_{1}$ with the condition that $g<1$. The value of $g$ depends on the turbulent boundary layer method used in the solver and will be determined later by trial and error. Using this in Eq.(8) and rearranging the terms, we have

$$
\Delta \theta=\theta_{e}\left(1-\frac{U_{e}}{U_{\infty}}\right)\left(\frac{U_{e}}{U_{\infty}}\left(g H_{1}-1\right)-1\right) .
$$

From Eqs.(7) and (10), we have,

$$
\theta_{\infty}=\theta_{e}+\Delta \theta=\theta_{e}\left(1+\left(1-\frac{U_{e}}{U_{\infty}}\right)\left(\frac{U_{e}}{U_{\infty}}\left(g H_{1}-1\right)-1\right)\right) .
$$


Once $\Delta \theta$ is calculated, it can be added to $\theta_{e}$ to obtain $\theta_{\infty}$ as shown in Eq.(11). Applying the Squire and Young correction to the end wake $\theta_{\infty}$ (one chord length downstream in XFOIL and RFOIL) will give us the value of momentum loss thickness at downstream infinity which can be used to calculate drag coefficient. The corrected value of the drag coefficient is given by,

$$
c_{d}=\frac{2\left[\theta_{\infty}\right]_{x=\infty}}{c}=\frac{2\left[\theta_{e}+\Delta \theta\right]_{x=\infty}}{c} .
$$

\section{Determination of Correction Factor $(g)$ for Mass Flow Shape Factor}

Eq.(10) gives an expression for the correction of the momentum thickness at the end of wake. This expression has an empirical parameter we need to determine, i.e., mass flow shape factor correction $(g)$. In order to determine the value of $g$, XFOIL results are used as the base line. A set of airfoils are analysed for a wide range of angle of attacks and the value of $g$ is varied to determine the value that resulted in good agreement with the experimental data for the drag. As a first guess, it is assumed that $g$ is a constant and not a function of some parameters. The correction factor $g$ is a consequence of the assumptions made in the boundary layer equations (described in section III ) and hence need not necessarily depend on the the boundary layer variables themselves, given that the boundary layer model remains the same when comparing two different solvers. At present, the analysis is confined to XFOIL and RFOIL only and the correction factor $(g)$ is related to the results from these two solvers.

The analysis to determine the value of $g$ was carried out by evaluating the drag coefficients of two airfoils, NACA 0012 and DU 97-W-300. The choice was made in order to have a value of $g$ which predicts drag well for thin airfoils as well as for thick airfoils. The analysis presented in this section is computed for incompressible flow conditions $\left(M_{\infty}=0\right)$ for conditions of natural transition with a free stream turbulence intensity of $0.07 \%\left(N_{\text {crit }}=9\right)$. For thin airfoils, there is not much of a problem in the predicted drag in the present model of XFOIL and RFOIL and thus the correction should not affect the results greatly. For thick airfoils, the under prediction in drag is noticeable and the correction is expected to improve the prediction. Preliminary results from XFOIL indicate the value of $g$ to be 0.4. Figures 3 and 4 show the predicted lift and drag characteristics for NACA 0012 and DU 97-W-300 airfoils for the case of the most recent XFOIL version, experimental data and XFOIL with the proposed drag correction using $g=0.4$.

The case of NACA 0012 is analysed as a benchmark case to make sure that the proposed correction does not affect drag for cases for which the XFOIL prediction is accepted to be accurate. As can be seen in figure 3, the drag correction improves the drag prediction and the curve moves closer to the curve with experimental data. The magnitude of the correction is comparable to the difference between the XFOIL prediction and the experimental data. The experimental data for NACA 0012 airfoil used for comparison was obtained from the airfoil data catalog by Miley. ${ }^{7}$

For the case of a thick airfoil without a significant trailing edge thickness, the drag prediction appears to improve by using the correction. Figure 4 shows the results for the DU $97-\mathrm{W}-300$ airfoil which is a $30 \%$ thick airfoil with a small trailing edge thickness $(\approx 1.8 \%$ of chord $)$. The corrected lift-drag polars are still not on top of experimental data as there is an over prediction of lift as well. This is due to the under prediction of $\delta^{*}$. The correction for drag deteriorates as the angle of attack becomes larger. In deep stall, the correction is no longer valid as the considered wake length of one chord is no longer sufficient to resolve a fully developed wake. In such cases, the edge velocity $U_{e}$ becomes higher than the free stream velocity and the drag correction then yields a negative value. However, this is due to the failure of the solver to predict the boundary layer parameters accurately in deep stall regions due to massive separation present in such a case. The experimental data for DU 97-W-300 airfoil used for comparison was obtained from the technical report by Timmer. ${ }^{8}$

At high angles of attack, the drag prediction deteriorates as the predicted drag with the correction is somewhat higher than experimental data. This can also be attributed to the overall drop in the accuracy of prediction in XFOIL due the strong adverse pressure gradients and trailing edge separation that is commonly observed near stall which can not be coped with by the strong-interaction method. The drag correction is sensitive to the change in the shape factor $H$ at the far-field wake location and proper care must be taken to refine the grid (mesh density) in order to obtain a reliable result. The value of $g=0.4$ is also verified in 


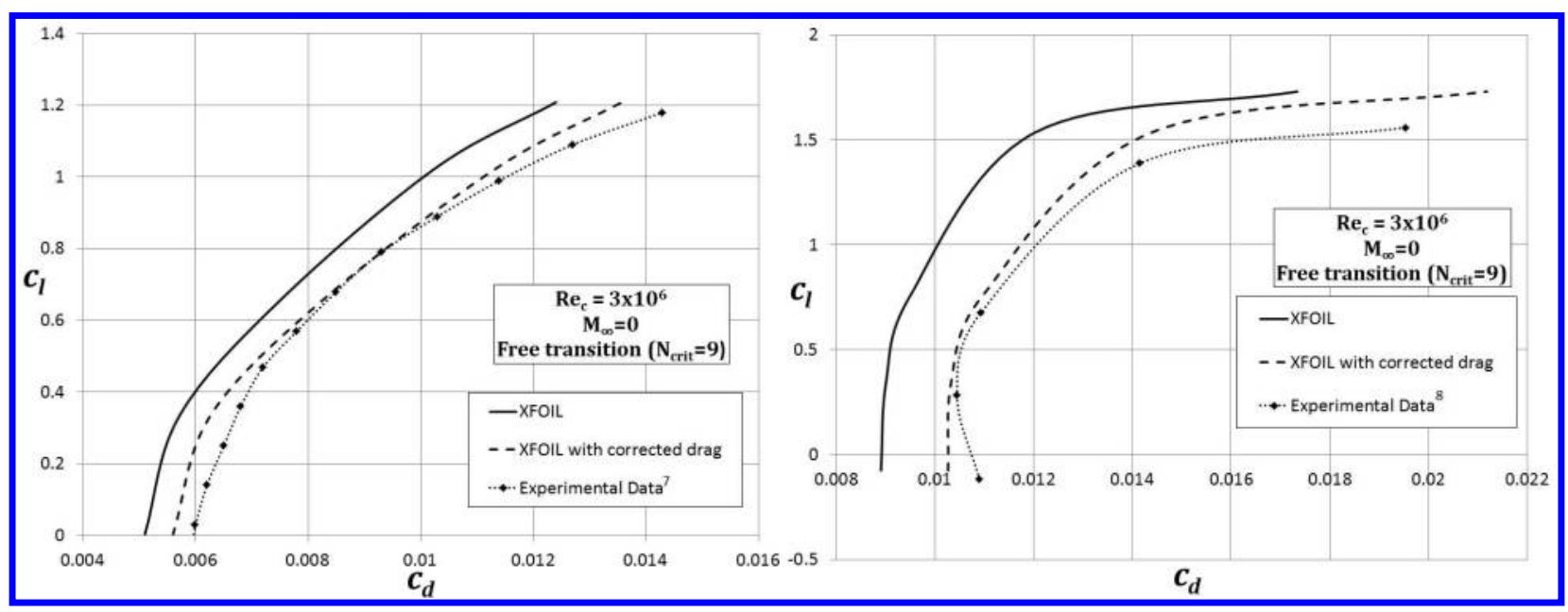

Figure 3: Comparison of Lift-Drag polars for NACA 0012

Figure 4: Comparison of Lift-Drag polars for DU $97-\mathrm{W}-300$

calculations for several other airfoils and the results are presented in section VI.

Once the value of the shape factor correction is established for XFOIL, the same procedure is repeated for RFOIL. For the case of RFOIL, $g=0.4$ causes an over prediction in drag. Figure 5 shows this behaviour for the case of the NACA 0012 airfoil. There is a noticeable over prediction in drag in RFOIL when the proposed drag correction formula is used. Since, the only empirical parameter in the drag correction formula is $g$, changing its value may improve the value of the predicted drag. The most significant difference between RFOIL and XFOIL is the $G-\beta$ equilibrium locus coefficients. Another difference is the quadrupling of the equilibrium shear stress level in the wake in RFOIL.

The above mentioned differences between the

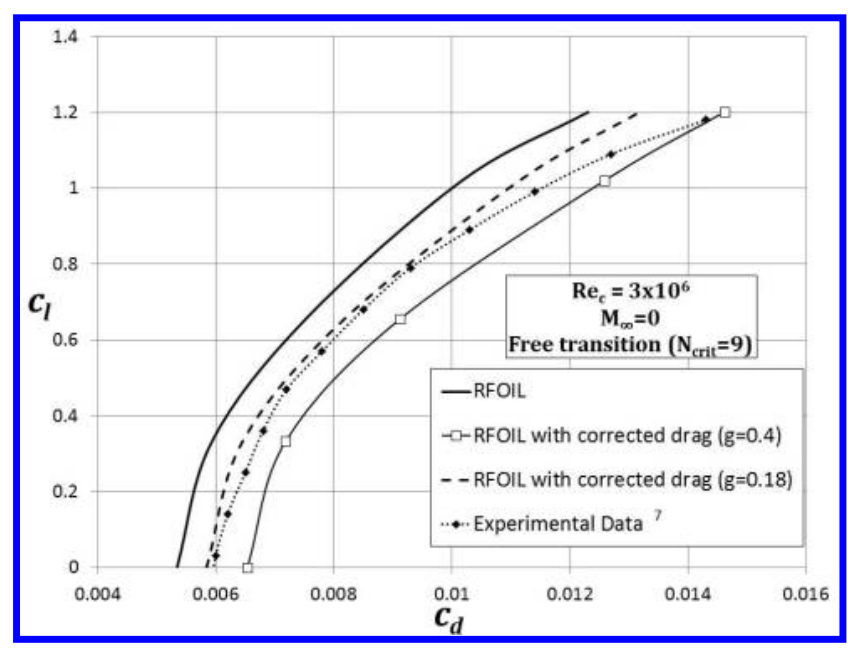

Figure 5: Predicted Lift-Drag polars for NACA 0012 in RFOIL formulation of RFOIL and that of XFOIL will certainly have an effect on the magnitude of the drag correction. This means the mass flow shape factor correction $g$ will no longer be a constant and needs to be related to the differences in the two models. In order to quantify the effect of these differences in the model and relate them to $g$, we need to look at how these differences affect the solution. The solution domain can be split into two parts, namely the airfoil surface and the wake. We will analyse the difference in the equilibrium shear stress levels in these two domains and then quantify the change as a weighted average of the equilibrium shear stress levels for the airfoil surface and that for the wake. The rationale behind such a weighted average is that the equilibrium shear stress levels are an indicator of the level of dissipation and the weighted average gives equivalent value of the change dissipation level in the whole domain.

On the airfoil surface, the only significant difference between XFOIL and RFOIL is in the $G-\beta$ equilibrium locus, namely the values of the coefficients $A$ and $B$. These coefficients affect the magnitude of the equilibrium 
shear stress. The equilibrium shear stress is described in the paper from Drela and Giles ${ }^{6}$ as,

$$
C_{\tau_{E Q}}=\frac{0.5 H^{*}\left(H_{k}-1\right)^{3}}{A^{2} B\left(1-U_{s}\right) H H_{k}^{2}}
$$

where the definitions of $H^{*}, H_{k}$ and $U_{s}$ can be found in the reference from Drela and Giles. ${ }^{6}$

In the above relation, the term $A^{2} B$ in the denominator is the most significant term that is different between RFOIL and XFOIL. Table 1 shows the values of the $G-\beta$ equilibrium locus coefficients $A$ and $B$ in XFOIL and RFOIL on the airfoil surface. Since the equilibrium locus is the most significant change between RFOIL and XFOIL, the difference in the equilibrium shear stress levels will play a major role in determining the level of error in momentum thickness. From Eq.(13), we have,

\begin{tabular}{|c|c|c|}
\hline Method & A & B \\
\hline \hline XFOIL & 6.7 & 0.75 \\
\hline RFOIL & 6.75 & 0.83 \\
\hline
\end{tabular}

Table 1: $G-\beta$ locus coefficients

$$
C_{\tau_{E Q}} \propto \frac{1}{A^{2} B},
$$

assuming that the rest of the terms do not have a significant dependence on $A$ and $B$. Comparing the equilibrium shear stress levels in XFOIL and RFOIL on the airfoil surface results in the following,

$$
\left[\frac{\left[C_{\tau_{E Q}}\right]_{R F O I L}}{\left[C_{\tau_{E Q}}\right]_{X F O I L}}\right]_{\text {airfoil }}=\frac{\left[C_{\tau_{E Q}}\right]_{R F O I L}}{\left[C_{\tau_{E Q}}\right]_{X F O I L}}=\frac{\left[A^{2} B\right]_{X F O I L}}{\left[A^{2} B\right]_{R F O I L}}=0.89 .
$$

In the wake, the equilibrium shear stress $C_{\tau_{E Q}}$ is quadrupled in RFOIL while it remains the same in XFOIL. Thus for the wake region, we have

$$
\left[\frac{\left[C_{\tau_{E Q}}\right]_{R F O I L}}{\left[C_{\tau_{E Q}}\right]_{X F O I L}}\right]_{\text {wake }}=\frac{4\left[C_{\tau_{E Q}}\right]_{R F O I L}}{\left[C_{\tau_{E Q}}\right]_{X F O I L}}=\frac{4\left[A^{2} B\right]_{X F O I L}}{\left[A^{2} B\right]_{R F O I L}}=3.56 .
$$

Thus, the effective ratio of the equilibrium shear stress predicted by RFOIL and that predicted by XFOIL can be taken as the weighted average of the equilibrium shear stress ratio on the airfoil surface and that on the wake. Thus, the ratio of the equilibrium shear stress in RFOIL and XFOIL is given by,

$$
\left[\frac{\left[C_{\tau_{E Q}}\right]_{\text {RFOIL }}}{\left[C_{\tau_{E Q}}\right]_{\text {XFOIL }}}\right]_{\text {effective }}=\frac{1}{2}\left(\left[\frac{\left[C_{\tau_{E Q}}\right]_{\text {RFOIL }}}{\left[C_{\tau_{E Q}}\right]_{\text {XFOIL }}}\right]_{\text {airfoil }}+\left[\frac{\left[C_{\tau_{E Q}}\right]_{\text {RFOIL }}}{\left[C_{\tau_{E Q}}\right]_{X F O I L}}\right]_{\text {wake }}\right)=2.225 .
$$

From the above equation we see that the equilibrium shear stress levels are higher in RFOIL which will result in a higher dissipation level. This means that the predicted value of $\theta_{e}$ will be higher in RFOIL and hence the correction in momentum thickness should be smaller in RFOIL compared to the one in XFOIL.

$$
[\Delta \theta]_{R F O I L}<[\Delta \theta]_{X F O I L}
$$

Since $\theta_{\infty}$ should be the same for an airfoil at a given angle of attack in both RFOIL and XFOIL, $\Delta \theta$ for XFOIL and RFOIL depends directly on the predicted value of $\theta_{e}$. RFOIL has a higher dissipation level (higher effective $C_{\tau_{E Q}}$ ) compared to that in XFOIL, resulting in a higher $\theta_{e}$, the correction $\Delta \theta$ should be inversely proportional to the effective $C_{\tau_{E Q}}$ level.

$$
C_{\tau_{E Q}} \propto \frac{1}{\Delta \theta}
$$

From Eq.(10), we can also infer

$$
\Delta \theta \propto g .
$$

Using Eqs.(17), (19) and (20), we have,

$$
\left[\frac{[\Delta \theta]_{\text {RFOIL }}}{[\Delta \theta]_{X F O I L}}\right]_{\text {effective }}=\frac{[g]_{R F O I L}}{[g]_{X F O I L}}=\left[\frac{\left[C_{\tau_{E Q}}\right]_{X F O I L}}{\left[C_{\tau_{E Q}}\right]_{\text {RFOIL }}}\right]_{\text {effective }}=\frac{1}{2.225} .
$$


Rearranging the terms and using the value of $g$ obtained for XFOIL results $(g=0.4)$ in the following,

$$
[g]_{R F O I L}=\frac{[g]_{X F O I L}}{2.225}=0.18 .
$$

Figure 5 shows the lift-drag polars using RFOIL for two values of $g$ for the case of the NACA 0012 airfoil. The value of $g=0.18$ yields a good agreement with the experimental data. An expression for the mass flow shape factor correction $g$ can be obtained by combining Eqs.(15),(16),(17),(21) and(22) resulting in the following ,

$$
[g]_{\text {anysolver }}=[g]_{X F O I L}\left(\frac{\left[A^{2} B\right]_{\text {anysolver }}}{\left[A^{2} B\right]_{X F O I L}}\right)\left(\frac{2}{1+x_{C_{\tau_{E Q}}}}\right),
$$

where $x_{C_{\tau_{E Q}}}$ is the equilibrium shear stress coefficient multiplier for the wake. Substituting the known values into the equation gives,

$$
[g]_{\text {anysolver }}=\frac{\left[A^{2} B\right]_{\text {anysolver }}}{42.084\left(1+x_{\tau_{\tau_{E Q}}}\right)} .
$$

Eq.(24) gives a generalised expression for the mass flow shape factor correction $(g)$ for any XFOIL like method. This methodology for evaluating the value of $g$ can be adopted for any XFOIL like viscous-inviscid interaction method which uses the integral boundary layer equations. The value of $x_{C_{\tau_{E Q}}}$ in RFOIL is 4 in accordance to the experimental results obtained by Narasimha and Prabhu, ${ }^{9}$ who suggested a quadrupling of the eddy viscosity in wake. The expression for drag coefficient including the proposed correction is as follows,

$$
c_{d}=\frac{2}{c}\left[\theta_{e}\left(1+\left(1-\frac{U_{e}}{U_{\infty}}\right)\left(\frac{U_{e}}{U_{\infty}}\left(\left(\frac{A^{2} B}{42.084\left(1+x_{C_{\tau_{E Q}}}\right)}\right) H_{1}-1\right)-1\right)\right)\right]_{x=\infty} .
$$

\section{Results}

The proposed drag correction method has been implemented in XFOIL and RFOIL and has been tested for a range of airfoils in order to verify the accuracy of drag prediction. There was a significant improvement observed for all the test cases considered. The analysis presented in this section is computed for incompressible flow conditions $\left(M_{\infty}=0\right)$ for conditions of natural transition with a free stream turbulence intensity of $0.07 \%\left(N_{\text {crit }}=9\right)$. Only for the case of the INNWIND.EU analysis (FFA-W3-301 airfoil), the free stream turbulence is set to $0.1 \%\left(N_{\text {crit }}=8.145\right)$.

Even for thinner airfoils, there is a noticeable improvement in drag prediction as seen from the case of NACA 0012. A similar trend is also observed for the NACA $63_{3}-418$ airfoil as seen in figure 6(a) and 6(b). For this airfoil, the drag under-prediction is approximately $12 \%$ at the minimum drag point when using the original XFOIL and RFOIL results. For thicker airfoils, the under-prediction in drag becomes more severe. The levels of under-prediction in drag can become as high as $30 \%$ for very thick airfoils such as DU 99-W-405LM which has a maximum thickness of 40.5\%. Figure 6 gives an illustration of the levels of drag under-prediction for thick wind turbine airfoils of varying thickness.

The drag correction yields excellent results in the linear lift regime although the drag in the post stall regime is not very well predicted. This is to be expected since the viscous-inviscid interaction method of XFOIL and RFOIL are not designed to handle massively separated flows.

For the AH 94-W-301 airfoil, the experimental and the predicted data (with improved drag) do not match satisfactorily. This is due to the over-prediction of lift observed in this case as shown in figure 7. For DU 99-W-405LM, the same issue of lift over-prediction exists although it is necessary to note that the experimental data used for this airfoil is synthesized from low Reynolds number airfoil data (using the ATG $^{10}$ tool). The ATG tool compares the geometry of any input airfoil to the airfoils in its database and interpolates the experimental data from the database to generate (synthesize) the aerodynamic coefficients of the input airfoil. Lack of open access experimental data on airfoils at higher Reynolds numbers is the reason why the synthesized data has been used in this case. The experimental data for Wortmann (FX series) and Althaus (AH series) airfoils was obtained from the University of Stuttgart. ${ }^{11}$ The experimental data for 


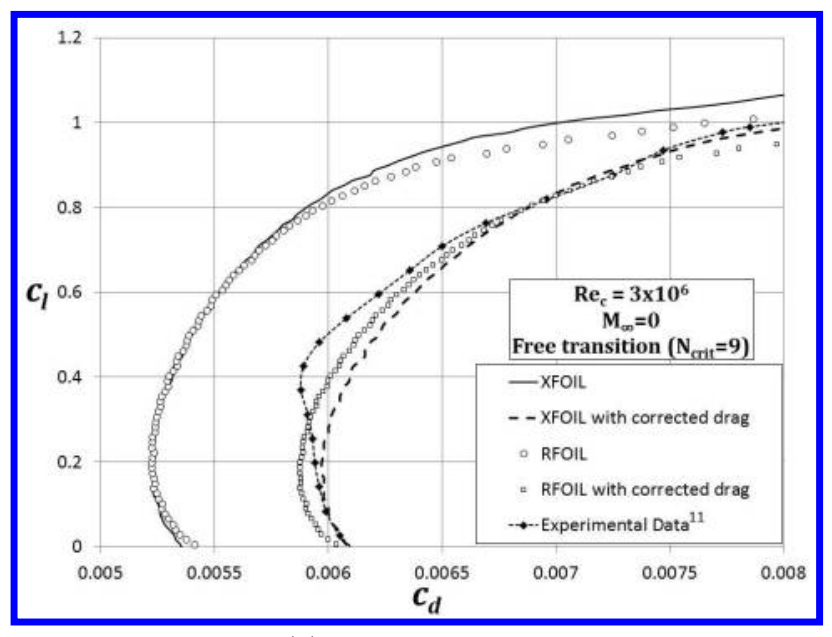

(a) $\mathrm{NACA} 63_{3}-418$

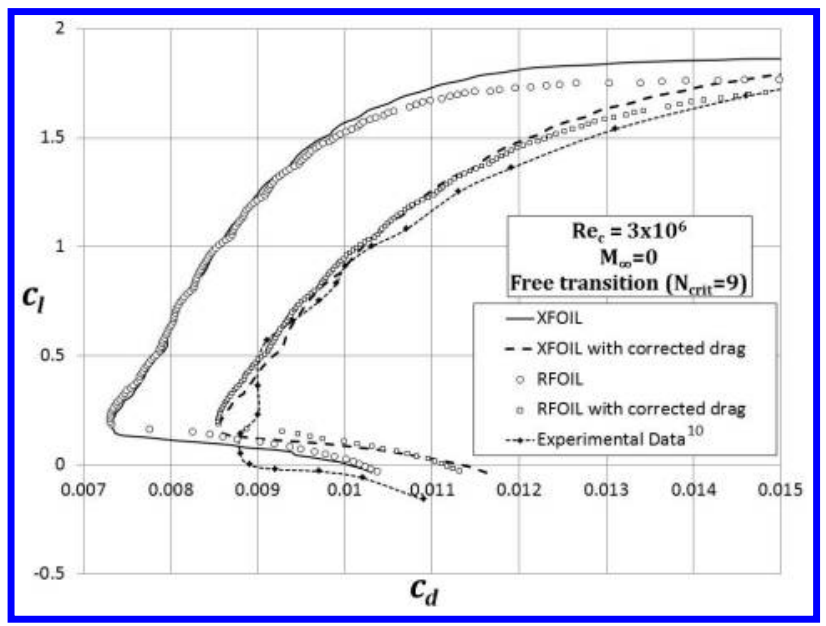

(c) FX $77-W-270 \mathrm{~S}$

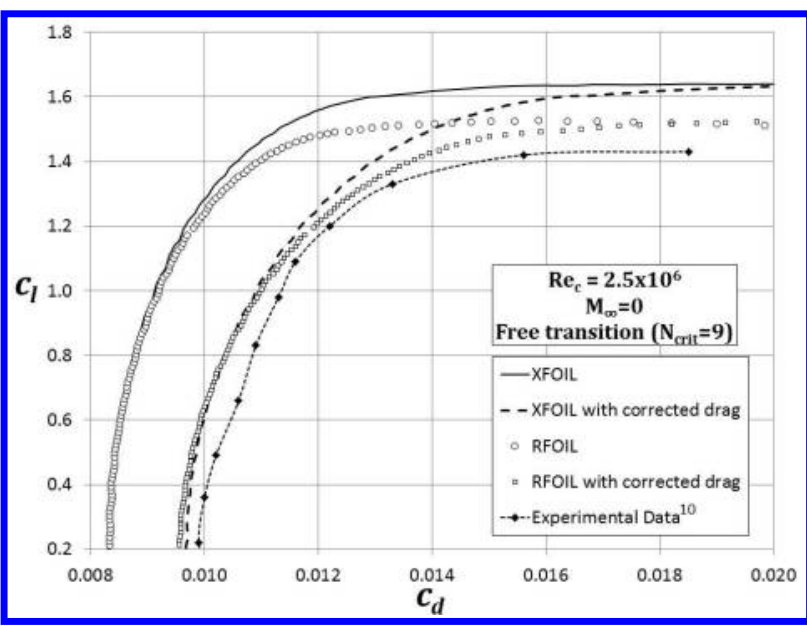

(e) AH 94-W-301

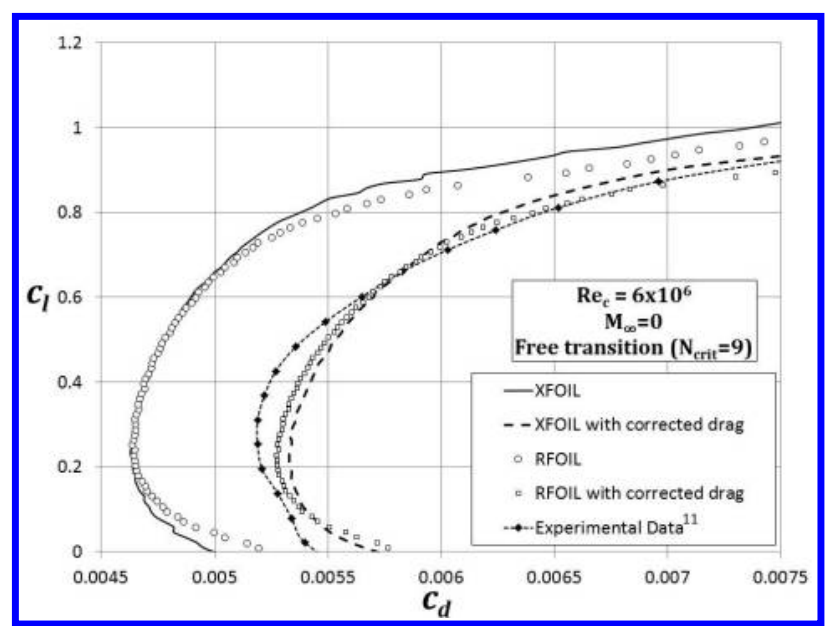

(b) [NACA $63_{3}-418$

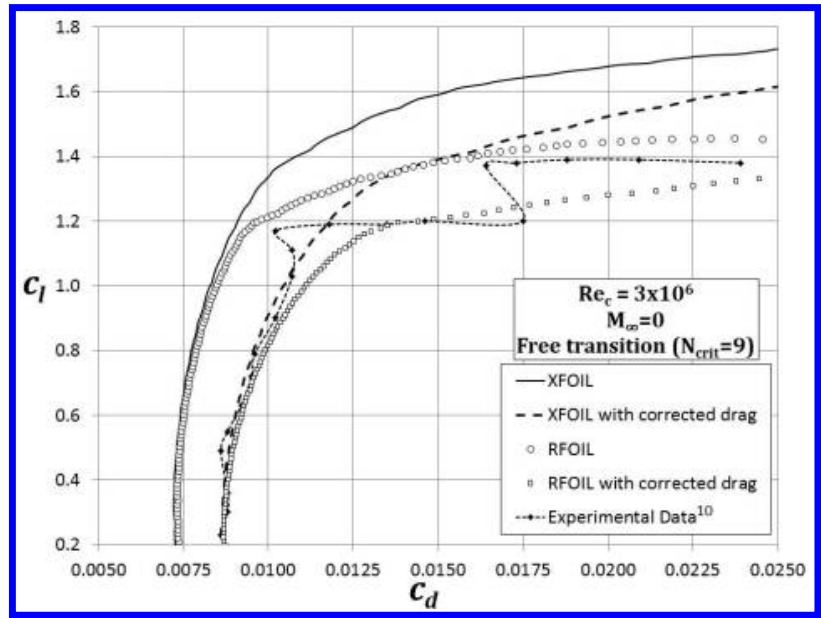

(d) FX 69-274

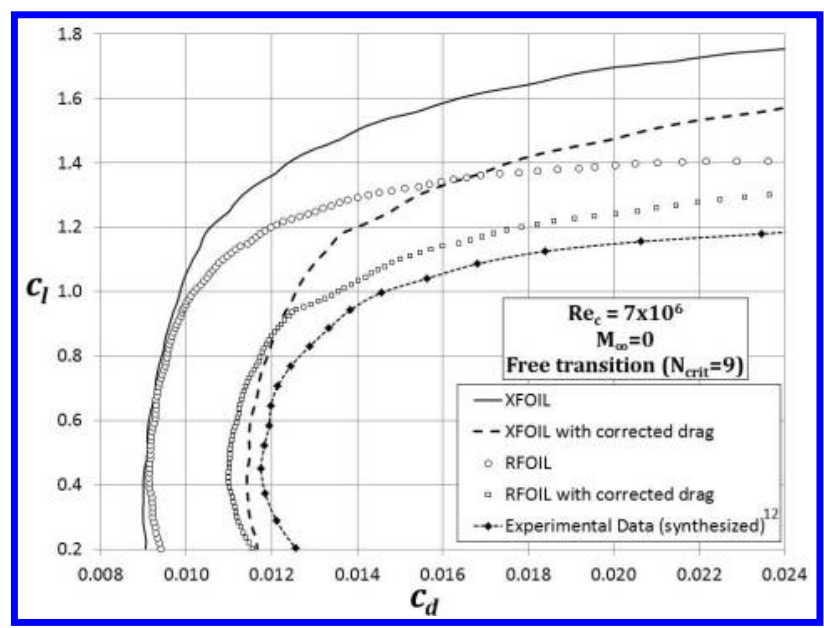

(f) DU 99-W-405LM

Figure 6: Comparison of Lift-Drag polars for thick wind turbine airfoils with and without the proposed drag correction to the experimental data 
NACA $63_{3}-418$ airfoil is obtained from Abbott \& von Doenhoff. ${ }^{12}$

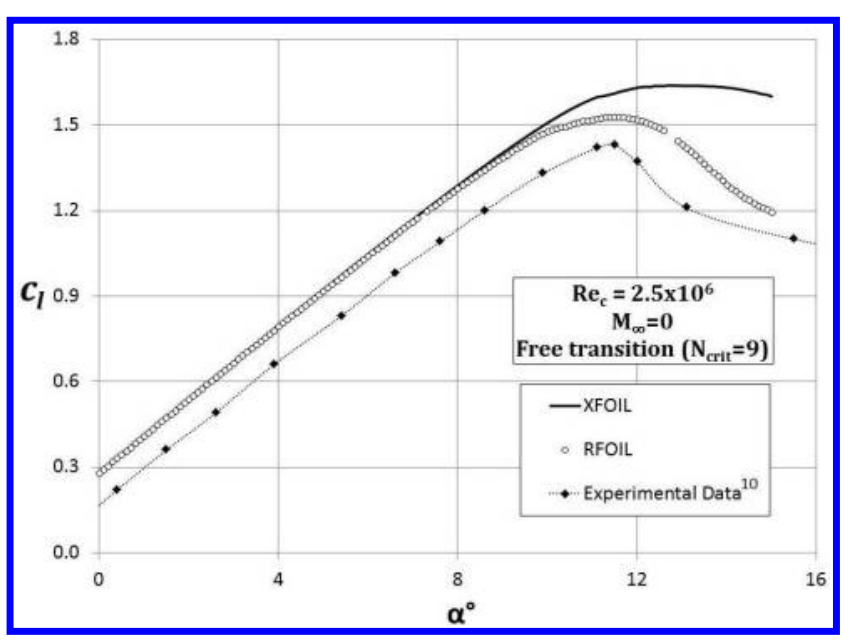

Figure 7: Predicted Lift curve for AH 94-W-301

The significance of the improvement in drag prediction using the developed correction can be realized by a comparison of the results of various commercial and in-house aerodynamic software with the results from the improved version of RFOIL. For this purpose, the FFA-W3-301 airfoil is chosen as this airfoil has been analysed using various solvers in the European project INNWIND.EU. ${ }^{13}$ The solvers used for computing the aerodynamic charecteristics of the selected airfoil are,

- EllipSys 2D : CFD software developed by DTU

- WMB (Wind Multi-Block): CFD software developed by CENER and University of Liverpool

- CFX : Commercial CFD software developed by ANSYS

A detailed description of these solvers and their analysis results can be found in INNWIND.EU deliverable report. ${ }^{13}$

The improved version of RFOIL performs better for both Reynolds numbers. For Reynolds number of $10 \times 10^{6}$, EllipSys2D over-predicts the drag coefficient. Drag coefficient usually decreases with increasing Reynolds numbers, while the values predicted by EllipSys2D at $10 \times 10^{6}$ Reynolds number are larger than the experimental values at $3 \times 10^{6}$.

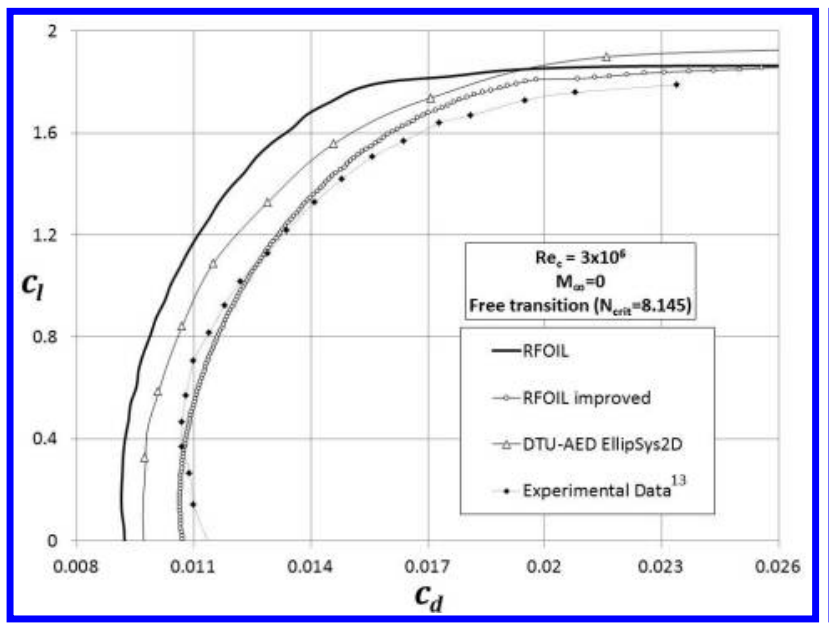

(a) Reynolds Number $=3 \times 10^{6}$

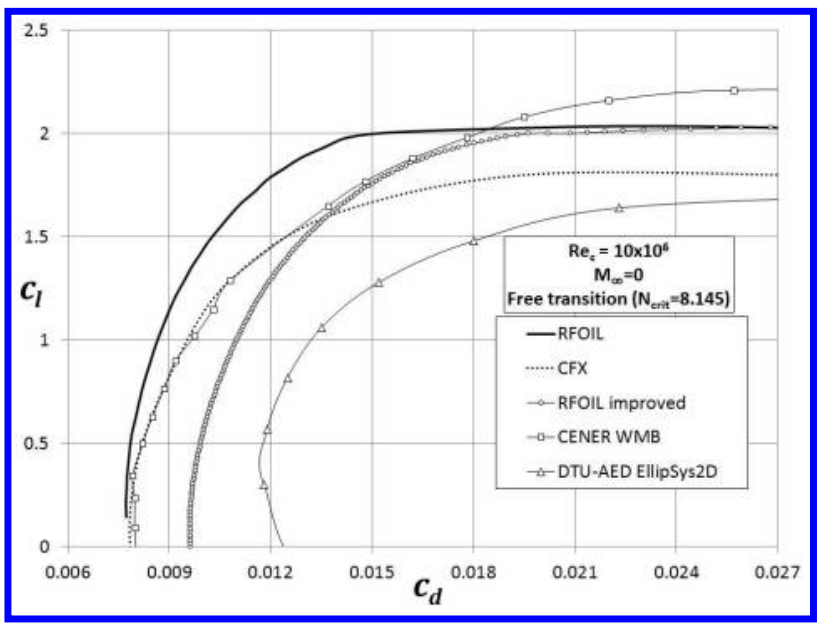

(b) Reynolds Number $=10 \times 10^{6}$

Figure 8: Comparison of Lift-Drag polars computed using various methods for FFA-W3-301 airfoil

\section{Conclusion}

To enable accurate prediction of drag for thick wind turbine airfoils, a modification to the existing drag calculation method in XFOIL-like methods has been developed. The general methodology of the correction is applicable to a wide range of viscous-inviscid interaction methods using a two-equation lag dissipation integral boundary layer method. The proposed method is applicable in regions of angle of attack prior to 
stall as the correction can become negative in deep stall region where at the end of the wake, the predicted value of velocity at the edge of the boundary layer exceeds the magnitude of the free stream velocity. This happens in the case of massively separated flows over the airfoil which is outside the range of applicability of these computational methods. The correction is applicable to airfoils with a relatively small trailing edge thickness $\left(h_{T E}<3 \%\right.$ of chord). Present analysis is performed for a wake length of one chord which is adequate for most airfoils. Increasing the wake length will require a recalibration of the value of the mass flow shape factor correction $(g)$ as the end wake values of $H_{1}$ will be quite high due to the rapid increase in $H_{1}$ at values of $H$ close to unity.

\section{Acknowledgments}

This work has been performed within the Design for Reliable Power Performance (D4REL) project granted by the Dutch Top Consortium for Knowledge and Innovation in Offshore Wind (TKI Wind op Zee) under number TKIW02007 and the AVATAR project, which is initiated by the European Energy Research Alliance (EERA) and carried out under the FP7 program of the European Union.

\section{References}

${ }^{1}$ Drela, M., "XFOIL: An analysis and design system for low Reynolds number airfoils," Low Reynolds number Aerodynamics, Springer, 1989, pp. 1-12.

${ }^{2}$ Rooij, R. P. J. O. M. Van, "Modification of the boundary layer calculation in RFOIL for an improved stall prediction," Tech. Rep. IW-96087R, TU Delft, 1996.

${ }^{3}$ Schlichting, H., Lecture series "Boundary Layer Theory", Part I - Laminar Flows, No. 1217, NACA, April 1949.

${ }^{4}$ Veldman, A. E. P., Boundary Layers in Fluids, University of Groningen, 2008, Lecture notes in Applied Mathematics.

${ }^{5}$ Green, J. E., Weeks, D. J., and Brooman, J. W. F., "Prediction of turbulent boundary layers and wakes in compressible flow by a lag-entrainment method," Tech. Rep. 3791, Aeronautical Research Council, 1977.

${ }^{6}$ Drela, M. and Giles, M. B., "Viscous-inviscid analysis of transonic and low Reynolds number airfoils," AIAA journal, Vol. 25, No. 10, 1987, pp. 1347-1355.

${ }^{7}$ Miley, S. J., "A catalog of low Reynolds number airfoil data for wind turbine applications," Texas A 6 M University, Vol. UC-60, No. RFP-3387, 1982

${ }^{8}$ Timmer, W. A., "Ontwerp en windtunneltest van profiel DU 97-W-300," Tech. Rep. IW-98003R, TU Delft, 1998, in Dutch.

$\checkmark{ }^{9}$ Narasimha, R. and Prabhu, A., "Equilibrium and relaxation in turbulent wakes," J.Fluid Mech., Vol. 54, 1972, pp. 1-17.

${ }^{10}$ Bot, E. T. G., "Aërodynamische Tabel Generator; Handleiding." Tech. Rep. ECN-C-01-077, Energy research Centre of the Netherlands, 2001, in Dutch.

${ }^{11}$ Althaus, D., "Stuttgarter Profilkatalog II: Niedriggeschwindigkeitsprofile," University of Stuttgart, 1996.

${ }^{12}$ Abbott, H. and von Doenhoff, A. E., "Measurements NACA in LTT-Langley," Tech. Rep. 422, NACA, 1949.

${ }^{13}$ Zahle, F., Riziotis, V., Madsen, H. A., and L.Bergami, "Benchmarked aerodynamic-structural design methods: Part B "Benchmarking of aerodynamic and aeroelastic models"," Tech. Rep. Deliverable D2.21, INNWIND.EU, 2013. 